\title{
Bloqueo epidural lumbar continuo para espasmos vesicales incoercibles
}

\author{
C. López Carballo, S. Vázquez del Valle, M. Garrido García, J. Pico Veloso, R. Valle Yáñez, \\ M. J. Bermúdez López y F. J. Pardo-Sobrino López \\ Servicio de Anestesiología, Reanimación y Tratamiento del Dolor. Hospital Lucus Augusti. Lugo
}

López Carballo C, Vázquez del Valle S, Garrido García M, Pico Veloso J, Valle Yáñez R, Bermúdez López MJ, Pardo-Sobrino López FJ. Bloqueo epidural lumbar continuo para espasmos vesicales incoercibles. Rev Soc Esp Dolor 2013; 20(2): 45-49.

\begin{abstract}
We present a clinical case of bladder spasms due to detrusor overactitivity, triggered by continuous vesicoclysis therapy, which was applied in a patient with benign prostatic hypertrophy-related hematuria. Bladder spasms turned out to be refractory to combined antimuscarinic, spasmolytic and systemic opioid therapies. Implantation of a lumbar epidural catheter was chosen for continuous epidural infusion of local anesthetics and opioid drugs as an alternate analgesic therapy, which provided the patient an optimum comfort, but let urethral indwelling catheterization and the maintenance of continuous vesicoclysis therapy as well.

We reviewed scientific literature concerning bladder-afferent neurotransmission blockade at epidural level for dysfunctional bladder pain therapy, and discussed several published theories about pain physiopathology and origin in those cases of dysfunctional bladder disturbance, with the aim to interpret the peculiarity and complexity of the described clinical case.
\end{abstract}

Key words: Blockade at epidural level. Dysfunctional bladder. Pain bladder. Overactive bladder syndrome. Neuromodulation.

Fuentes de financiación: ninguna.

Conflicto de interés: ninguno declarado.

Recibido: 12-03-12.

Aceptado: 21-05-12.

\section{RESUMEN}

Presentamos un caso clínico de espasmos vesicales por hiperactividad del detrusor de la vejiga, desencadenados por lavado vesical continuo aplicado en un paciente con hematuria, en el contexto de hipertrofia benigna de próstata. Los espasmos llegaron a ser refractarios a tratamiento sistémico con antimuscarínicos, espasmolíticos y opioides. Se optó por la colocación de un catéter epidural lumbar para infusión continua de anestésicos locales y opioides como terapia analgésica alternativa, que no solo proporcionó un aceptable grado de confort, sino que permitió el mantenimiento del sondaje uretral y el lavado vesical continuo.

Realizamos una revisión bibliográfica sobre el uso del bloqueo epidural de la neurotransmisión de las aferencias sensitivas vesicales en dolor de origen disfuncional vesical, y analizamos algunas de la teorías publicadas sobre la fisiopatología y génesis de dolor en estos trastornos vesicales, con la intención de interpretar las peculiaridades y complejidad del dolor en el cuadro del caso presentado.

Palabras clave: Bloqueo epidural. Disfunción vesical. Dolor vesical. Síndrome de vejiga hiperactiva. Neuromodulación.

\section{INTRODUCCIÓN}

Desde el punto de vista urológico existe una serie de síndromes con disfunción del tracto urinario inferior, que se solapan entre sí: síndrome de vejiga hiperactiva, cistitis intersticial, síndrome de vejiga dolorosa, síndrome de urgencia-frecuencia miccional y síndrome de dolor pélvico crónico (1-3).

Los trastornos disfuncionales vesicales crónicos generan una pérdida de calidad de vida y morbilidad por sintomatología de tenesmo, urgencia miccional, poliaquiuria, incontinencia, etc. y potencialmente predisponen a la aparición 
Rev. Soc. Esp. del Dolor, Vol. 20, N. ${ }^{\circ}$ 2, Marzo-Abril 2013

de dolor vesical. Probablemente en la génesis de este dolor contribuyen no solo la hiperactividad miogénica funcional vesical, sino también fenómenos inflamatorios vesicales (4) y fenómenos neurogénicos de sensibilización central secundarios $(1,2,5)$.

A diferencia de los espasmos vesicales que pueden aparecer en el postoperatorio de cirugía urológica, en las obstrucciones agudas del tracto urinario inferior o en algunos tumores infiltrantes vesicales, los espasmos vesicales por síndrome de vejiga hiperactiva surgen dentro de un contexto de una regulación al alza de las señales aferentes sensitivas del tracto urinario inferior, fruto de una neuroplasticidad desarrollada de forma crónica y en paralelo a la disfunción vesical, que precisa ser tratada de forma específica. La hiperactividad aferente sensitiva vesical puede provocar que ciertos estímulos aplicados sobre el urotelio vesical, que en condiciones normales serían escasamente nociceptivos, se transmitan a centros de procesamiento sensorial de forma masiva, desencadenando respuestas de hiperactividad vesical con dolor.

\section{CASO CLÍNICO}

Presentamos un caso clínico de disfunción vesical crónica (hiperactividad del detrusor), asociada a dolor intenso refractario, secundario a espasmos vesicales, en paciente con necesidad de lavado continuo vesical.

Varón de 58 años que ingresa en el hospital por cuadro de hematuria macroscópica, acompañada de dolor suprapúbico, portador de sonda uretral desde $72 \mathrm{~h}$ antes del ingreso por retención aguda de orina. Entre sus antecedentes personales destacan una lumbociatalgia derecha por protusiones discales lumbares, urolitiasis remota tratada con litotricia extracorpórea y un síndrome miccional crónico de larga evolución, en el contexto de una hipertrofia benigna de próstata según biopsia prostática, a tratamiento crónico con tamsulosina por vía oral (vo), tras dos resecciones transuretrales prostáticas previas. El paciente ingresa a cargo del Servicio de Urología en el hospital, siendo sometido a estudio con técnicas de imagen. En la ecografía abdominopélvica se objetivaron signos de "vejiga de lucha", sin datos de uropatía obstructiva, y la tomografía computarizada abdominopélvica con contraste reveló hipertrofia prostática y engrosamiento parietal difuso vesical, compatible con hipertrofia del detrusor. Se procede a cambio de sonda uretral por una sonda adaptada a lavado vesical continuo, para disolución de coágulos intravesicales. Durante la instauración del lavado vesical aparecen episodios de dolor suprapúbico tipo cólico, acompañados de intenso cortejo neurovegetativo y reflujo de líquido vesical hematúrico, contra gravedad, del suero de lavado vesical, situado unos sesenta centímetros sobre el nivel paciente. Tras descartar problemas obstructivos y de posicionamiento de la sonda uretral, se diagnostica de espasmos vesicales por hiperactividad del detrusor y se inicia tratamiento con butilbromuro de hioscina, metamizol y dexketoprofeno intravenosos (iv), tolterodina y alprazolam, ambos por vo, y cloruro mórfico subcutáneo sin éxito. Dada la persistencia de la clínica dolorosa y la intolerancia a la terapia de lavado vesical, se decide la colocación de un catéter epidural lumbar en el espacio intervertebral L3-L4, por el que se administra un bolo de $80 \mathrm{mg}$ de lidocaína al $2 \%$ con $50 \mu \mathrm{g}$ de fentanilo y posteriormente se inicia una perfusión de levobupivacaína al $0,125 \%$ con fentanilo, a razón de $5 \mathrm{mg} /$ hora y $4 \mu \mathrm{g} /$ hora respectivamente. Tras la instauración del bloqueo epidural se consigue un excelente control de sintomatología dolorosa y de las manifestaciones urodinámicas de espasmo vesical, con un nivel de bloqueo sensitivo a nivel del dermatoma T12 y con un bloqueo motor en grado 0 en la escala de Bromage. Se mantiene con la citada perfusión hasta la explantación accidental del catéter epidural, que ocurre siete días más tarde, iniciándose analgesia iv con cloruro mórfico a $0,96 \mathrm{mg} / \mathrm{hora}$. Al décimo día de ingreso se interviene de forma programada bajo anestesia intradural, para adenomectomía prostática retropúbica, según técnica de Millin, manteniéndose como analgesia postoperatoria la pauta de cloruro mórfico iv, asociado a paracetamol iv. Así mismo se mantuvo la tolterodina vo. A las $48 \mathrm{~h}$ de postoperatorio, y todavía bajo terapia de lavados continuos vesicales, reaparecen los episodios de espasmos vesicales dolorosos, de similares características a los previos, precisando aumentar el ritmo de la infusión de cloruro mórfico hasta 1,8 mg/hora. Se descarta la colocación de nuevo catéter epidural, por heparinización profiláctica postoperatoria. Tras la retirada del lavado vesical continuo postoperatorio por cese de la hematuria, el cuadro clínico desaparece completamente. El paciente refirió un mayor alivio del dolor con el bloqueo epidural, respecto a la combinación terapéutica de espasmolíticos, cloruro mórfico y tolterodina.

\section{DISCUSIÓN}

La hiperactividad del detrusor consiste en contracciones involuntarias vesicales durante la fase de llenado cistométrico, con o sin incontinencia urinaria acompañante (6) y es una de las múltiples causas de síndrome de vejiga hiperactiva (7). Entre las causas que producen hiperactividad del detrusor destacan la obstrucción del tracto urinario inferior, las causas neurogénicas, los casos de debut postcorrección de incontinencia urinaria con cabestrillos y las causas idiopáticas (6). El síndrome de vejiga hiperactiva engloba, aparte de la hiperactividad del detrusor, otras patologías que producen una disregulación de las señales inhibitorias y excitatorias del reflejo de la micción, produciendo sintomatología de urgencia miccional, con o sin 
incontinencia urinaria, con poliuria y nocturia asociadas frecuentemente (7). Cuando se añade dolor vesical, de más de seis meses de evolución, a la sintomatología de síndrome de vejiga hiperactiva se denomina síndrome de vejiga dolorosa $(1,2,5)$. En los casos de síndrome de vejiga dolorosa, en los que se documenta por medio de cistoscopia úlceras de Hunner en el urotelio vesical, esta entidad pasa a denominarse cistitis intersticial $(3,8)$. En la práctica clínica la delimitación de cada uno de estos trastornos vesicales es poco nítida. Actualmente algunos autores empiezan a considerar todas estas patologías con disfunción vesical y sintomatología del tracto urinario inferior, como son el síndrome de vejiga hiperactiva, la cistitis intersticial/síndrome de vejiga dolorosa, la prostatitis crónica y el dolor pélvico crónico, como un continuum sindrómico del mismo proceso fisiopatológico de hiperactividad aferente sensitiva vesical, inflamación y sensibilización central, con solapamiento y presentación de síntomas variables (1-3,5). De hecho, con frecuencia pacientes diagnosticados y tratados inicialmente como casos de síndrome de vejiga hiperactiva acaban desarrollando un síndrome de vejiga dolorosa/ cistitis intersticial (2).

La vejiga es el órgano pélvico con mayor densidad de inervación sensorial, lo cual la convierte en un órgano potencialmente vulnerable para la génesis de dolor pélvico y disfunción vesical (1). El urotelio vesical actúa como punto de inicio de la neurotransmisión aferente vesical de múltiples estímulos hacia el sistema nervioso central, en donde se desencadenan respuestas tales como dolor, el reflejo de control voluntario de la micción o reflejos cardiovasculares de origen vesical $(3,4,9)$. Las terminales sensitivas vesicales principalmente implicadas en este proceso de neurotransmisión son fibras tipo A $\delta$ y C. Las fibras sensitivas $A \delta$, que se localizan entre las capas del músculo detrusor, actúan fundamental como mecanorreceptores, activándose con la distensión vesical provocada por el almacenamiento de orina en la vejiga (6). Las fibras sensitivas $\mathrm{C}$ se localizan tanto en el urotelio como en la capa submucosa vesical y están en su mayor parte en estado inactivo (1). Las fibras $\mathrm{C}$ pasan a estado activo frente a estímulos químicos en la composición de la orina (urea, potasio, contaminantes de la orina en infecciones, etc.), o bien estímulos térmicos y estímulos mecánicos de distensión urotelial a través de vías purinérgicas (9) y vaniloides $(3,9,10)$. El urotelio está recubierto de forma fisiológica por una capa de glucosaminoglucanos, la cual parece proteger también a estas fibras sensitivas $\mathrm{C}$ frente a estos estímulos (11). En múltiples patologías vesicales inflamatorias crónicas, tales como infecciones urinarias, la cistitis actínica o la cistitis intersticial/síndrome de vejiga dolorosa, la capa de glucosaminoglucanos parece deteriorarse exponiendo el urotelio a estímulos que activan las fibras $\mathrm{C}$ y que desencadenan fenómenos inflamatorios uroteliales $(1,4,6)$. Las fibras $\mathrm{C}$ al activarse liberarían taquiquininas y sustancia $\mathrm{P}$, que actuarían como mediadores inflamatorios, activando mastocitos y la cascada inflamatoria, y al mismo tiempo actuarían como mediadores nociceptivos y posiblemente neurotróficos, reclutando a un creciente número de fibras C, lo cual parece explicar la disfunción vesical y dolor que aparece en ciertas patologías vesicales inflamatorias crónicas $(2,3)$. Por otra parte, en el síndrome de vejiga hiperactiva, considerada como trastorno fundamentalmente funcional vesical, se han encontrado un incremento de los receptores muscarínicos tipo M2 y M3 uroteliales (12), así como un incremento en la excreción urinaria de factor de crecimiento nervioso $(2,3,12)$, por lo que se especula que existe una regulación al alza de la activación nerviosa vesical en este trastorno urodinámico. Esta regulación al alza de la aferencias nerviosas vesicales, en el síndrome de vejiga hiperactiva, parece ser la responsable de la hiperactividad y/o hipertrofia del detrusor y podría predisponer a la aparición de fenómenos de hiperalgesia e incluso alodinia vesical, en el caso de llegar a desarrollar fenómenos inflamatorios uroteliales $(1,3,5)$. En estudios con modelos experimentales animales se ha documentado incluso una sensibilización nociceptiva cruzada entre diversos órganos pélvicos, lo que hace suponer que, ciertos trastornos inflamatorios crónicos son capaces de inducir una hiperactividad sensitiva neuronal en órganos pélvicos vecinos (2). En la práctica clínica esta sensibilización cruzada pélvica explicaría la asociación con cierta frecuencia de trastornos disfuncionales y dolorosos pélvicos, lo cual a veces dificulta el manejo tanto diagnóstico como terapéutico de los mismos $(1,3)$. De este modo el síndrome de vejiga dolorosa/cistitis intersticial puede asociarse a dolor pélvico de múltiples etiologías, como la prostatitis crónica, vulvodinia, endometriosis, disfunción del suelo pélvico y síndrome de intestino irritable $(1-3,5,11,13)$. La sensibilización pélvica cruzada podría explicar además el fenómeno por el cual la sintomatología de dolor y disfunción urinaria puede reaparecer en casos de cistitis intersticial tratados con técnicas de denervación vesical, lo cual fue interpretado previamente por algunos autores como un fenómeno de reinervación (14). El hecho de que en la práctica clínica sea posible tratar con bloqueos epidurales y bloqueos simpáticos lumbares el dolor y la disfunción miccional, producido por la cistitis intersticial, ha conducido a algunos autores a comparar este trastorno urinario con un síndrome de dolor regional complejo $(14,15)$. Si tenemos en cuenta todos estos hechos en conjunto, entonces parece cobrar fuerza la teoría que considera a todos estos trastornos de disfunción dolorosa vesical como un síndrome de dolor visceral complejo, que implica inflamación crónica neurógena, hiperactividad aferente primaria y sensibilización central $(1,2,5)$.

El caso que exponemos solo puede ser etiquetado de disfunción vesical crónica (síndrome de vejiga hiperactiva por hiperactividad del detrusor), secundaria a obstrucción 
del tracto urinario inferior por hipertrofia prostática, ya que carece de criterios cistoscópicos y de duración del dolor, para poder hacer el diagnóstico de síndrome de vejiga dolorosa/cistitis intersticial. La hiperactividad vesical en estos casos de obstrucción se genera como mecanismo compensador para vencer la resistencia al vaciado vesical. En el caso que presentamos, a pesar de disminuir la obstrucción del tracto urinario inferior con sondaje uretral permeable, se mantuvo la hiperactividad vesical. Tanto el sondaje uretral como el lavado vesical pueden llegar a actuar como estímulos nociceptivos y de descarga vesical, incluso en vejigas no hiperactivas, pero no llegan a desencadenar espasmos vesicales de semejante magnitud urodinámica como en los casos de hiperactividad del detrusor. Por tanto creemos que el caso presentado ilustra un ejemplo de procesamiento sensorial aberrante, asentado sobre una disfunción vesical crónica por hiperactividad del detrusor. La aplicación del bloqueo epidural en el caso que presentamos se justifica por el hecho de que la estimulación vesical, creada por el lavado vesical continuo, actuó como un estímulo térmico y traumático nociceptivo, así como de estímulo de descarga vesical por hidrodistensión vesical, el cual resultaba clínicamente insoportable para el paciente. Probablemente la cateterización uretral previa contribuyó como agente lesivo urotelial favorecedor de inflamación. La desaparición mantenida de los espasmos vesicales tras la explantación del catéter epidural y la posterior reaparición de los mismos, dos días después del bloqueo intradural quirúrgico, es compatible, desde el punto de vista fisiopatológico, con fenómenos de regulación a la baja y posteriormente al alza de las señales aferentes vesicales provocadas respectivamente por los bloqueos neuroaxiales y su cese de acción.

Los fármacos antagonistas de receptores muscarínicos se consideran actualmente el tratamiento de primera línea para los síntomas de hiperactividad del detrusor $(6,16)$, ya que parece que son capaces de modular la actividad aferente vesical tanto a nivel de las fibras $\mathrm{C}$ como en las $\mathrm{A}-\delta$, y disminuir los niveles urinarios de factor de crecimiento nervioso (12), no obstante pueden presentar efectos adversos (2) y su eficacia puede verse disminuida con su uso prolongado. En el caso que presentamos, sin embargo, la tolterodina no fue capaz de prevenir la reaparición de espasmos vesicales en el postoperatorio tras el cese de la acción de los bloqueos neuroaxiales. La utilización del bloqueo epidural lumbar para bloquear las aferencias sensitivas en trastornos funcionales y dolorosos vesicales, como en el caso que presentamos, no es algo novedoso. Sin embargo las publicaciones científicas que hemos revisado corresponden exclusivamente todas a casos documentados de cistitis intersticiales. Irwin y cols. (12) publicaron una serie de trece casos de cistitis intersticial tratados con infiltraciones epidurales periódicas de bupivacaína, con una tasa de alivio de dolor, durante más de 24 horas, del 75\%, asociado a una mejoría en la calidad de vida y del sueño. Estos autores describen en su serie de pacientes dos casos como no respondedores y otros dos casos con pérdida de eficacia del bloqueo en sucesivas sesiones de infiltración epidural. Takatani y cols. (13) publicaron un caso de cistitis intersticial en el que aplicaron secuencialmente bloqueos epidurales periódicos, bloqueo del plexo hipogástrico y finalmente gabapentina vo. Una consideración que hay que tener en cuenta en los resultados obtenidos por estos autores es que los bloqueos epidurales no eran continuos, sino infiltraciones epidurales periódicas. La regulación al alza progresiva de las aferencias sensitivas vesicales en estos trastornos disfuncionales probablemente sea uno de los factores que contribuya a desarrollar taquifilaxia frente a los bloqueos epidurales periódicos. La potencial diferencia de efectividad clínica del bloqueo epidural continuo prolongado respecto a bloqueos epidurales periódicos en este contexto clínico no ha sido comparada con estudios de investigación. Doi y cols. (15) publicaron dos casos de cistitis intersticial, tratados con bloqueos epidurales continuos con éxito, en términos de alivio de dolor vesical y decremento de síntomas miccionales, a los que finalmente aplicaron sendos bloqueos simpáticos lumbares con agentes neurolíticos. Sukiennik y cols. (17) realizaron mediciones de excreción urinaria de sustancia $\mathrm{P}$ por radioinmunoanálisis, en una serie de cinco casos de cistitis intersticial tratados con bloqueos continuos epidurales de tres días de duración. Estos autores encontraron una excreción urinaria elevada de la sustancia $\mathrm{P}$, con un aumento inicial de los niveles basales de este péptido tras inicio del bloqueo epidural lumbar, seguido de un descenso posterior de sus niveles. Este hallazgo hizo hipotetizar a estos autores que el bloqueo epidural continuo podría inducir una depleción de sustancia $\mathrm{P}$ en las aferencias sensitivas vesicales (17). Sin embargo el potencial efecto terapéutico del bloqueo epidural continuo, aplicado en los trastornos con disfunción vesical crónica, no ha sido evaluado con estudios científicos de mayor calidad. Por este motivo y siguiendo los criterios de la medicina basada en la evidencia, el bloqueo epidural en este contexto clínico alcanza el grado C de recomendación, con un nivel de evidencia 3, y está considerado como una medida analgésica temporal para crisis de dolor intratable (8), a pesar de mejorar también los síntomas de disfunción vesical $(14,15)$. El riesgo de retención urinaria, secundaria al bloqueo epidural, disminuye con el uso de concentraciones bajas de anestésico local, siendo posible su aplicación en algunos de estos pacientes sin necesidad de sondaje vesical (17), e incluso en el caso del bloqueo epidural periódico en régimen de paciente ambulatorio (14).

El diagnóstico y tratamiento etiológico de la causa que produce la regulación al alza de las aferencias vesicales (desobstrucción del tracto urinario inferior, reeducación 
del suelo pélvico, restauración de la capa de glucosaminoglucanos uroteliales, neuromodulación sacra, etc.) resulta fundamental para corregir la disfunción urinaria y evitar el desarrollo progresivo de dolor vesical. Esta premisa resulta difícil de llevar a cabo en los casos idiopáticos y neurogénicos de estas disfunciones vesicales, en donde se han aplicado diversos fármacos, entre ellos algunos con capacidad neuromoduladora como la amitriptilina, gabapentina, duloxetina o la toxina botulínica (8). La disfunción vesical, altamente discapacitante y refractaria, puede ser una indicación de técnicas de neuroestimulación eléctrica (neuroestimulación aferente de Stoller -18-, neuromodulación sacra -5,16- y neuromodulación del nervio pudendo -19-), que suelen mejorar la disfunción vesical y secundariamente el dolor de origen vesical.

\section{CONCLUSIÓN}

El bloqueo epidural continuo constituye una terapia puente efectiva, previa a la aplicación de tratamientos etiológicos más específicos en las disfunciones vesicales crónicas con gran discapacidad y dolor asociados. Se necesitan todavía estudios científicos de calidad para profundizar en el conocimiento de estos trastornos vesicales crónicos y del potencial papel terapéutico del bloqueo epidural aplicado en los mismos.

\section{CORRESPONDENCIA:}

Carlos López Carballo

Servicio de Anestesiología, Reanimación y Tratamiento del Dolor

Hospital Lucus Augusti

San Cibrao s/n (27003) Lugo.

e-mail: carlos.lopez.carballo@sergas.es

\section{BIBLIOGRAFÍA}

1. Chung MK, Butrick CW, Chung CW. The overlap of interstitial cystitis/painful bladder syndrome and overactive bladder. JSLS 2010;14:83-90.

2. Saini R, Gonzalez RR, Te AE. Chronic pelvic pain syndrome and the overactive bladder: the inflammatory link. Curr Urol Rep 2008,9:314-9.

3. Sant GR: Etiology, pathogenesis and diagnosis of interstitial cystitis. Rev Urol 2002;4(Suppl 1):S9-S15.
4. Damiano R, Cicione A. The role of sodium chondroitin sulphate in the management of bladder disease. Ther Adv Urol 2011;3(5):223-32.

5. Fariello JY, Witmore K. Sacral neuromodulation stimulation for IC/PBS, chronic pelvic pain and sexual dysfunction. Int Urogynecol J. 2010;21:1553-8.

6. Ashok K, Wang A. Detrusor overactivity: an overview. Arch Gynecol Obstet 2010;282:33-41.

7. Prieto L, Castro D, Esteban M, et al. Estudio epidemiológico descriptivo del diagnóstico de hiperactividad del detrusor en las unidades de diagnóstico urodinámico en España. Actas Urol Esp. 2011. doi:10.1016/j.acuro.2011.06.015.

8. Fall M, Oberpenning F, Peeker R. Treatment of bladder pain syndrome/interstitial cystitis 2008: Can we make evidencebased decisions? Eur Urol 2008;54:65-78.

9. Andersson K-E, Gratzke C, Hedlund P. The role of the transient receptor potential (TRP) superfamily of cation-selective channels in the management of the overactive bladder. BJU Int 2010;106:1114-27.

10. Juszczak K, Ziomber A, Thor PJ. Effect of partial and complete blockade of vanilloid (TRPV1-6) and ankyrin (TRPA1) transient receptor potential ion channels on urinary bladder motor activity in an experimental hyperosmolar overactive bladder model. J Physiol Pharmacol 2011;62(3):321-6.

11. Kahn BS, Stanford EJ, Mishell DR, et al. Management of patients with chronic pelvic pain of bladder origin: a consensus report. Curr Med Res Opin 2005;21(4):509-16.

12. Liu H-T, Chancellor MB, Kuo H-C: Decrease of urinary nerve growth factor levels after antimuscarinic therapy in patients with overactive bladder. BJU Int 2009;103:166872.

13. Takatani J, Takeshima N, Okuda K, et al: A case of perineal pain related to interstitial cystitis which was supposed to be relieved with gabapentin. J Anesth 2009;23:474-5.

14. Irwin PP, Hammonds WD, Galloway TM. Lumbar epidural blockade for management of pain in interstitial cystitis. BJU 1993;71:413-6.

15. Doi K, Saito Y, Nikai T, et al. Lumbar sympathetic block for pain relief in two patient with interstitial cystitis. Reg Anesth Pain Med 2001;26(3):271-3.

16. Thompson JH, Sutherland SE, Siegel SW. Sacral neuromodulation: therapy evolution. Indian J Urol 2010;26(3):379-84.

17. Sukiennik A, Carr DB, Bonney I, et al. The effect of short -term epidural local anesthetic blockade on urinary levels of substance P in interstitial Cystitis. Anesth Analg 2004; 98(3):846-50.

18. Karademir K, Baykal K, Sen B, et al. A peripheric neurostimulation technique for curing detrusor overactivity: Stoller afferent neurostimulation. Scan J Urol Nephrol 2005; 39:230-3.

19. Peters KM, Killinger KA, Boguslawski BM, et al. Chronic pudendal neuromodulation: expanding available treatment options for refractory urologic symptoms. Neurol Urodyn 2010;29(7):1267-71. 